\title{
Molecular interaction and inhibitory potential of polyphenol on dna repair pathway in small cell lung cancer: a computational study
}

\begin{abstract}
Poly (ADP) ribose polymerase-1 (parp-1) is a ubiquitous and transient nucleo-protein with supra-function in orchestrating DNA damage repair symphony and has prognostic value in small cell lung cancer (SCLC). Accumulating evidence indicate that the substrate $\left(\mathrm{NAD}^{+}\right)$ binding catalytic domain (active site) of this protein launches its activation. However, blocking this binding site is the key node in the inhibition of DNA repair pathway, thus signifying its importance in the control of small cell lung cancer progression. Approved drugs for the treatment of disease burden may stop the growth of the tumor cells by blocking some of the enzymes needed for cell growth or by killing the cells, stopping them from dividing via combinatorial efficacy. Clinical tractability of this strategy has been proven to potentially promote undesirable side effect. In the current study, our aim therefore, was to outsource a best in class small molecule inhibitor of PARP-1 from healthfriendly source. We therefore, employed computational tools to investigate the therapeutic relevance of compounds from natural source as parp-1 inhibitor via molecular docking approach. 30 phyto-compounds were retrieved from the selected plant, $+(-)$ gallocatechin GC (a polyphenolic compound) showed best binding affinity with the catalytic domain (active site) of parp-1 on comparism to the standard inhibitors. GC interact with the amino acid residue around the $4 \AA \hat{~}$ of the catalytic domain (ASN-868, ASN-767, SER-864, GLY863, HIS-862, TYR-896, PHE-897, TRP-861, ALA-898, SER-904, TYR-907, LYS-903) as evaluated by energy decomposition per residue of GC-parp-1 complex. The result from this investigation thus, project GC as a lead compound which may inhibit PARP-1 as a single therapy in the treatment of small cell lung cancer.
\end{abstract}

Keywords: enzymes, protein, pathogenesis, nicotinamide, autoparylation
Volume 6 Issue 3 - 2017

\author{
Olumide K Inyang, Olaposi I Omotuyi, \\ Adewale J Ogunleye, Gabriel O Eniafe, \\ Bamidele Adewumi, Damilohun S Metibemu \\ Centre for Bio computing and Drug Development,Adekunle \\ Ajasin University, Nigeria
}

Correspondence: Olumide K Inyang, Centre for Bio computing and Drug Development, Adekunle Ajasin University, Nigeria, Email I20401065@aaua.edu.ng

Received:September 08, 2017 | Published: October 25, 2017
Abbreviations: SSB, single-stranded breaks; DSB, double stranded breaks; DNA; ADME, absorption, distribution, metabolism and excretion; $\mathrm{MW}$, molecular weights; $\mathrm{HBD}$, hydrogen bond donors; HBA, hydrogen bond acceptors; FSU, co-crystalized inhibitor; GC, polyphenol compound; SCLC, small cell lung cancer

\section{Introduction}

Small cell lung cancer (SCLC), is a highly aggressive pulmonary neuroendocrine neoplasm which represents about $20 \%$ of all lung carcinoma ${ }^{1}$ cases with an ever increasing proliferative index and an unusually strong predilection towards metastasis., ${ }^{2,3}$ Despite the unceasing basic and clinical research that started about 30 years ${ }^{4}$ no sustainable improvements have been established in the treatment of this pulmonary insult. Human SCLC is currently ranked as the leading cause of cancer-death worldwide with annual mortality rate. However, several attempts have been geared at understanding the molecular mechanisms that underpin the pathogenesis of human SCLC.

In recent time, SCLC has been characterized by the aberrant expression of number of genes implicated in DNA damage repair pathway such as PARP-1. Wealth of studies has vetted PARP-1 as a critical biochemical therapeutic target and was confirmed by reverse phase protein array (RPPA) method, as one of the overexpressed proteins involved in DNA repair pathway in large SCLC proteomic profiling in cell lines. ${ }_{5}$ PARP-1 is a chromatin- associated enzyme localized in the nucleus, ${ }^{6-9}$ programmed to orchestrate DNA damage resolution symphony, especially in the context of base excision repair (BER) during single-strand (SSB), and double-strand break (DSB) repair, this activation process is a survival mechanism. It play singlehanded role as an E2F1 co-activator, ${ }^{10-11}$ this prompt its inhibition may have biphasic action, with direct effects on DNA repair process and in concert with other E2F1-regulated DNA repair proteins. PARP-1 capacity to associate with DNA is established via direct binding to ERK2 which potently stimulates it enzymatic activity and, as a result, increases PARP-1 autoPARylation. PARP-1 activation promotes the ERK2-dependent phosphorylation of a downstream effector (DNAbinding transcription factor Elk1) resulting in an increase in histone acetylation and target gene expression. ${ }^{12}$ In concert, an NAD ${ }^{+}$synthase [nicotinamide mononucleotide adenylyltransferase-1 (NMNAT-1)] associates with PARP1, thus allowing for a proximal source of $\mathrm{NAD}^{+}$ cofactor and increasing PARP-1 activity.

Automodification of PARP-1 transfer signal activation to effector pathways by recruiting repair protein complexes, which may either directly throw in, DNA repair programs or coordinate repair via chromatin unfolding. ${ }^{13}$ In response to damage, PARP-1 recognises the damage, binds to the injury site and recruit PARP-dependent DNA damage repair proteins to the site such as XRCC1, DNA ligase III, and DNA polymerase $\beta(\operatorname{pol} \beta)$ to mediate DNA damage repair. ${ }^{14-18}$ Finally, PARP-1 and the repair complex dissociate from the DNA for a cycling and the single-stranded break is repaired leading to cell survival and the tumour cell growth continues.

Pharmacologic inhibition of PARP-1 by nascent novels in cancer drug arsenal is the strategy against single-stranded breaks (SSB) and double stranded breaks (DSB) repair machinery by the downregulation of key components of the homologous recombination pathway and 
which eventually abrogate tumor growth ${ }^{5}$ or making the tumour cell more susceptible to chemotherapy. In presence of parp inhibitor, PARP-1 is still recruited to break site, but can no longer activate parp-dependent DNA repair proteins because it catalytic activity can no longer be powered by $\mathrm{NAD}^{+}$binding. Therefore, PARP1 remain bound to the DNA, stalling the replication fork to DNA replication. This stalling drives DSBs formation ${ }^{19-21}$ and homologous recombination is impaired which eventually leads to cell death and the tumour cell progression is abrogated.

Previous chemotherapeutic strategies targeting PARP-1 solely rely on combinatorial efficacy. Alternatively here, our approach was used based on a singly acting bioactive compounds derived from natural sources to inhibit this candidate protein (PARP-1). Phytopharmaceticals from natural source acting as chemoprotective agent serve as alternative and safer cancer treatments and constitute the major source of current available anti-cancer drugs. In this study, we employ in-silico approaches which provide a high-quality interaction between the ligand and receptor. The bioactive compounds from green tea were screened for by their binding affinities against of PARP-1 catalytic domain to elucidate their anti-tumour effect to overcome tumour cell progression and proliferation by blocking the protein via conformational change which may alter the biological function of the implicated target (PARP-1) in this disease burden. Four lead compounds retrieved from above procedures are then channelled to Lipinski rule of five $^{22}$ on ADMET (Adsorption, Distribution, Metabolism, Excretion and Toxicity) properties summarized in Figure 1. GC was able to fulfill the rule of five on ADMET properties. Finally we demonstrated that GC may inhibit PARP-1 leading to abrogation of SCLC progression as a safe and single therapy Figure 2.

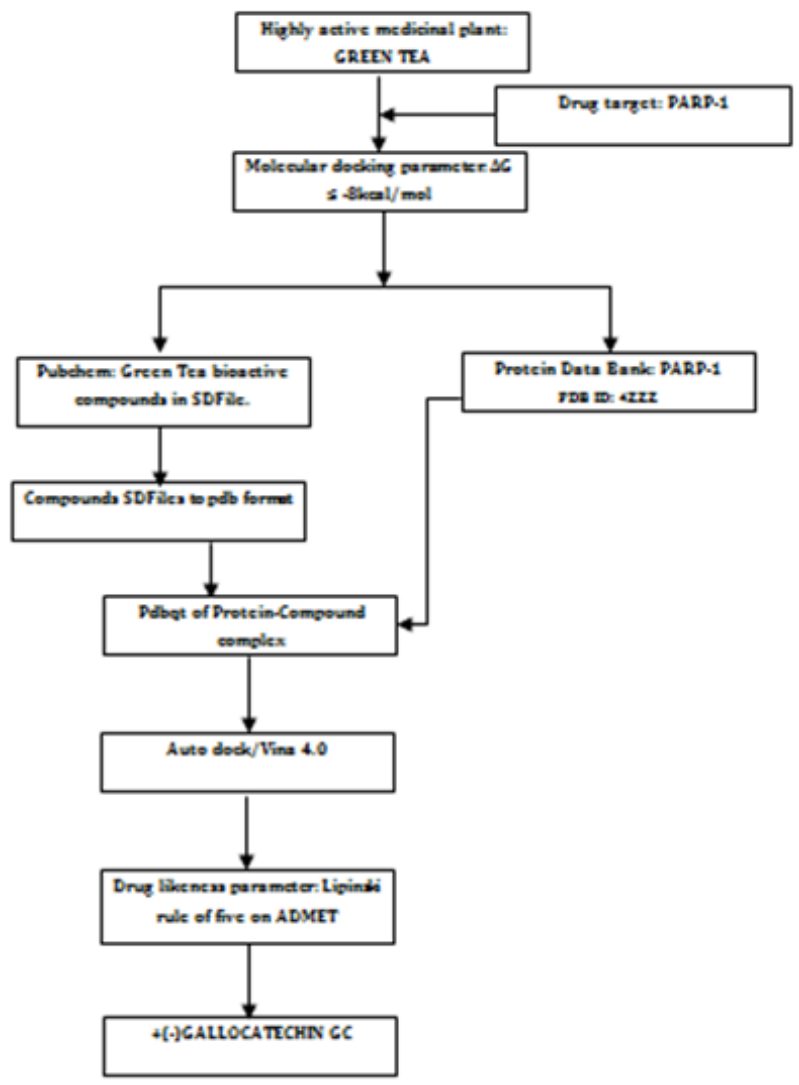

Figure I The modus operandi used to limit the number of compounds involves two main limiting steps: First limitation was done on the basis of binding energy and the second using drug-likeness parameters.
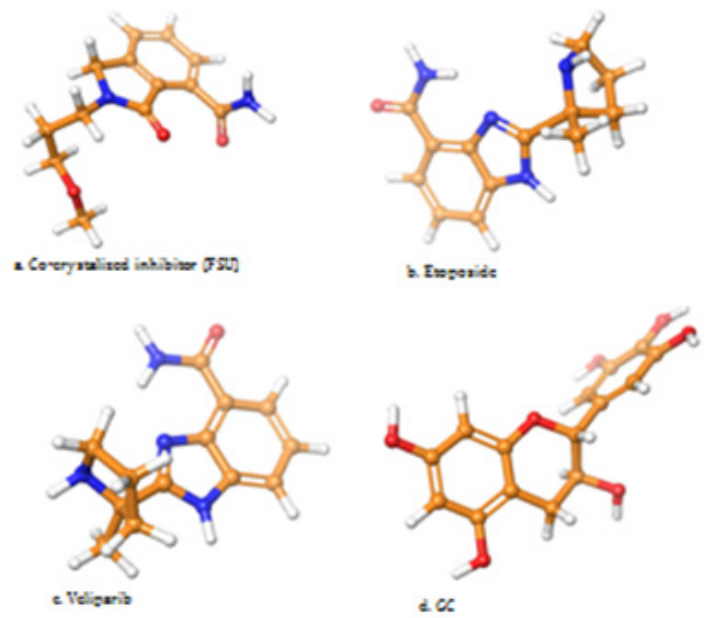

Figure 2 3D structure of lead compound (d) GC and established drugs (a) Co-crystalized inhibitor (FSU), (b) Etoposide and (c) Veliparib.

\section{Materials and methods}

\section{Target preparation}

Molecular docking were employed to the X-ray crystallography structure of human poly ADP-ribosyl polymerase-1 (PARP-1) catalytic domain bound to as isoindolinone inhibitor (FSU) (PDB ID: 4ZZZ) was retrieved from the protein data bank (http://www.rcsb. org). The structure visualized using the molecular graphics program PyMol ${ }^{\circledR}$ intended for the structural visualization of proteins. Water and ligand coordinates were deleted prior to the molecular docking.

\section{Ligand preparation}

Two dimensional structure of compounds (phytoligands) in green tea was retrieved from the PubChem ${ }^{\circledR}$ database. Optimized ligand was docked into distinguished model using Ligand Fit theory in the Auto Dock 4.2.

\section{Docking protocol}

Molecular docking protocols are widely used for predicting the binding affinities for a number of ligands. In current work, our aim was to examine the possibility of molecular interaction between the experimental bioactivities of the inhibitor under study and compounds from the natural source by their scoring binding conformation. In order to get accurate results, the docking experiment was performed with the default parameters. The protein was treated as a rigid body, ${ }^{23}$ while the rotatable bonds of the ligands were set to be free. The precalculated grid maps at the size set at 60,60 , and $60 \mathrm{~A}^{\circ}(\mathrm{x}, \mathrm{y}$, and $\mathrm{z})$ to include all the amino acid residues. The spacing between grid points was 0.375 angstroms. The time to dock one ligand was approximately 1-2 min. Docking using AutoDock/Vina, was performed on a hp workstation (Z800) with an Intel Pentium D processor $(3.06 \mathrm{GHz})$ and Tesla k40 GPU.

\section{ADME screening}

ADME (Absorption, Distribution, Metabolism and Excretion) screening for the polyphenolic compounds was done using available online server (http://www.scfbio-iitd.res.in/). ADME screening helps in detecting drug likeliness of compounds. According to Lipinski's rule of five, the number of rotatable bond, compound molecular weights 
(MW), calculated logarithm of the partition coefficient between n-octanol and water $(C \log P)$, molar refractivity, number of hydrogen bond acceptors (HBA) and number of hydrogen bond donors (HBD) were used to assess the "drug-likeness" (Lipinski, 2004).

Table I Showing Etoposide, Veliparib, and GC binding energies obtained from docking with PARP-I

\begin{tabular}{|c|c|c|c|c|}
\hline $\begin{array}{l}\text { Compound } \\
\text { Name }\end{array}$ & $\begin{array}{l}\text { Binding Energy } \\
\text { (Kcal/mol) }\end{array}$ & $\begin{array}{l}\text { No of } \\
\text { H-Bond }\end{array}$ & $\begin{array}{l}\text { Residues Involved by } \\
\text { Hydrogen Bonding }\end{array}$ & Amino Acid Residues found Within $4 \AA \hat{~}$ \\
\hline Etoposide & -11.1 & 4 & $\begin{array}{l}\text { ARG-878,ARG-878, MET-890, } \\
\text { TYR-907 }\end{array}$ & $\begin{array}{l}\text { ASP-770, ASP-768, ILE-872, ARG-878, ILE-879, } \\
\text { GLU-763, TYR-889, MET-890, LYS-903, GLU- } \\
\text { 988, ALA-898, SER-904, PHE-897, TRP-86I, } \\
\text { HIS-862, GLY-863,TRP-907, SER-864, ILE-872, } \\
\text { ARG878, LEU-877, ILE-895 }\end{array}$ \\
\hline Veliparib & -9.2 & 3 & TRP-86I, GLY-863,ALA-898 & $\begin{array}{l}\text { ASN-767, GLU-763,TRY-907, SER-864, SER- } \\
904 \text { GLY-863,TRP-86I,ALA-898, PHE-897, } \\
\text { TYR-896, HIS-862, }\end{array}$ \\
\hline $\begin{array}{l}\text { Isoindolinone } \\
\text { inhibitor(FSU) }\end{array}$ & -8.8 & 4 & $\begin{array}{l}\text { HIS-862, GLY-863, ASN-868, } \\
\text { SER-904, }\end{array}$ & $\begin{array}{l}\text { ASN-868, ASN-767, SER-864, GLY-863, HIS- } \\
\text { 862,TYR-896, PHE-897,TRP-86I,ALA-898, } \\
\text { SER-904,TYR-907, LYS-903 }\end{array}$ \\
\hline GC & -11.6 & 6 & $\begin{array}{l}\text { PHE-897, SER-904, SER-864,ASN- } \\
\text { 767,ASP-770, GLN-759 }\end{array}$ & $\begin{array}{l}\text { ASN-868, ASN-767, GLN-759, SER-864,TYR- } \\
\text { 907, GLY-863, HIS-862, SER-904, PHE-897, } \\
\text { TYR-86I, LYS-903, ALA-898,TYR-889 }\end{array}$ \\
\hline
\end{tabular}

Table 2 ADME result for the established drugs and polyphenol compound on the rule of five formulations

\begin{tabular}{llllll}
\hline Compound & Molecular Weight & CLogP & HBD & HBA & Molar Refractivity \\
\hline Etoposide & 638 & 0.6 & 5 & 13 & 0 \\
Isoindolinone inhib (FSU) & 246 & -0.21 & 1 & 3 & 57.57 \\
GC & 312 & 2.37 & 2 & 7 & 74.72 \\
Veliparib & 248 & 1.63 & 3 & 1 & 69.42 \\
\hline
\end{tabular}

HBA: Hydrogen Bond Acceptor; HBD: Hydrogen Bond Donor; CLogP:The Logarithm of the Partition Coefficient between n-octanol and Water.

\section{Hydrogen and $\pi$-stacking analysis}

The two-dimensional representation of molecules is a popular communication medium in chemistry and the associated scientific fields. Computational methods for drawing small molecules with and without manual investigation are well-established and widely spread in terms of numerous software tools. Concerning the planar depiction of molecular complexes, there are considerable less choice. We employ Proteinsplus, an online server (http://proteinplus.zbh. uni-hamburg.de) which automatically generates two-dimensional diagrams of marcromolecular complexes, showing the ligand, interactions, and interacting residues. ${ }^{24,25} \mathrm{We}$ complexed the FSU and $\mathrm{GC}$ with the protein separately in pdb format using Pymol and then submitted it on the sever page.

\section{Results}

The current study features computational approach to elucidate the molecular interaction, binding mode and inhibitory potential of selected polyphenolic compound of medicinal plant by the procedures displayed in Figure 1 and selected FDA-approved inhibitors on PARP-1 SCLC. Table 1 shows the binding energy and order the order of chemical interaction of natural compound (GC) in comparison to Etoposide, Veliparib and co-crystalized inhibitor (FSU) within the PARP-1 catalytic domain. The binding mode of the compounds and the interacting amino acids around $4 \AA$ of human PARP-1 is given in Figure 3a-d while the amino acid residues involved in hydrogen bonding networking and $\pi$-stacking interaction between PARP-1 and GC, PARP-1 and co-crystalized inhibitor (FSU) are presented in Figure 5. The docking study was performed using AutoDock 4.2 with PyMol tool whereas Figure 6 displayed the bar chart of the binding energy. The poses snapshot displayed in Figure 4 was done using PyMol. Molecular docking helps in studying the molecular interactions between ligand molecules and target protein macromolecule prior to possible in vitro analysis. Protein structural analysis and ADME assessment were performed on available web server. Human PARP-1 was retrieved from PDB database with PDB ID: 4ZZZ, and use as target for docking simulation. The ligands used in this study were retrieved form Pubchem database in sdf format and prepared for docking simulation. The computed ADME results for the compounds are given in Table 2 and 3D structures of all compounds aforementioned are displayed in Figure 2.

\section{Discussion}

Poly (ADP) ribose polymerase-1 belong to PARP family of enzymes. This enzyme has been known for its DNA damage resolution mechanism via poly ADP-ribosylation of proteins involved in DNA repair pathway during DNA damage. It is therefore logical to surmise that inhibiting PARP-1-dependent DSB/SSB repair, represents a sound pharmacological approach.

Here, we identify GC from green tea as a potent inhibitor of PARP1. Our first strategy was to perform a high throughput PARP-1 based screening to select compounds with favourable binding kinetics. The authenticity of our observations were rightly guided in the light of previously described PARP-1 inhibitors (cocrystalized Isolindolinone Inhib (FSU), Etoposide and Veliparib). The established drugs used in the treatment of extensive stage PARP-1 derived SCLC were docked into the active site (catalytic domain) of PARP-1 with the Gibbs free energy $(-8.8 \mathrm{Kcal} / \mathrm{mol},-11.2 \mathrm{Kcal} / \mathrm{mol},-9.2 \mathrm{Kcal} / \mathrm{mol})$ respectively. However, docking of the compounds from the natural source against PARP-1 active site using the same grid center coordinate, the compounds that with higher Gibbs free energy above the established drugs were selected, catechin $-11.7 \mathrm{Kcal} / \mathrm{mol}$, (-)-epigallocatechin $-11.2 \mathrm{Kcal} / \mathrm{mol}$, GC $-11.6 \mathrm{Kcal} / \mathrm{mol}$, quercetin $-11.5 \mathrm{Kcal} / \mathrm{mol}$, Theaflavin $-12.3 \mathrm{Kcal} / \mathrm{mol}$. We then deployed Lipinski rule of 5 to all these selected compounds and the established drugs. Excitingly, GC with Gibb's free energy $-11.6 \mathrm{Kcal} / \mathrm{mol}$ was able to fulfil Lipinski rule of five based on ADMET properties listed in Table 2. 
Protein-ligand interaction play a fundamental role in structuralbased drug design. In a typical PARP-1 antagonism scenario, clinically relevant inhibition is unambiguously decided by a combo of 12 amino acid residues located within $4 \AA$ hydrogen bonding distance in the enzyme's active site. The interacting amino acid residues within $4 \AA$ that participate in stabilizing the protein-ligand complex were (ASN-868, ASN-767, GLN-759, SER-864, TRY907, GLY-863, HIS-862, SER-904, PHE-897, TYR-861, LYS-903, ALA-898) and they are the main contributors to the stabilization of protein-ligand complex formed by PARP-1 and GC (Table 1 \& Figure 3a). To resolve the order of chemical bonds, we studied the binding network of previously described PARP-1 inhibitors and proposed them as benchmarks for efficacious PARP-1 antagonism. It is evident from Figure 5, the polyphenolic compound form hydrogen bonding network with one of the reported critical amino acid residue (SER904), indispensable for the nicotinamide binding pocket of the enzyme ${ }^{26}$ and hence competitively block the catalytic activity. Hydrogen has a major role in structural stability of many biological molecules and enzyme catalysis. Other molecular interaction between PARP-1 and the phenolic compounds include $\pi-\pi$ interaction with the phenolic backbone, hydrophobic and electrostatic attractions. From Figure 5, it is clear that a planarly-oriented $\pi-\pi$ stack coupled with a hydrophobic patch which putatively exists between PARP-1 derived TYR907 (an aromatic ring amino acid) and GC aromatic ring. These two are crucial requisite for overcoming possible hydration shell energy and ligand desolvation within the binding pocket. ${ }^{26}$ From Figure $4 \mathrm{c}$, the polyphenol compound (GC) efficiently occupies the hydrophobic pocket $(\mathrm{AD} \text { site })^{27}$ in an orientation that favours interaction with adjacently positioned but inhibition deciding roles. Most series of PARP-1 inhibitors take the advantage of this spacious pocket to improve solubility, potency and other pharmacologic properties.

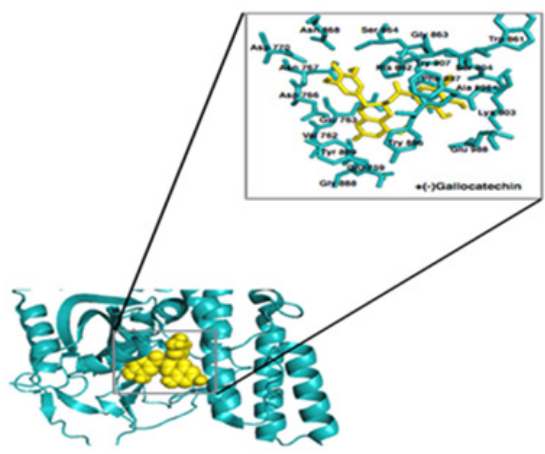

Figure 3a Molecular interaction between +(-)gallocatechin and human PARPI.

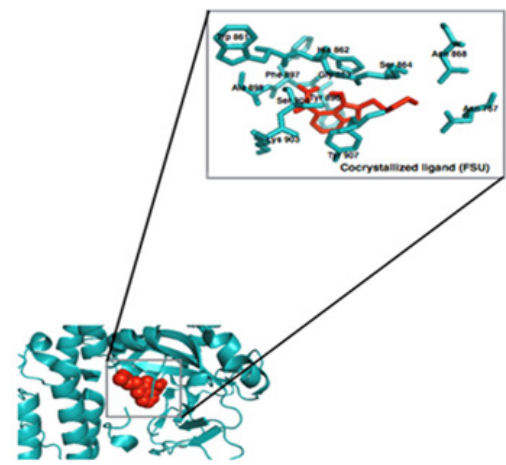

Figure $3 \mathbf{b}$ Molecular interaction between co-crystalized ligand (FSU) and human PARP-I.

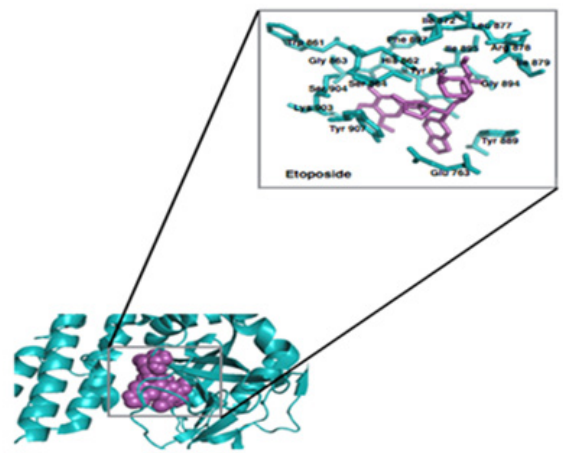

Figure 3c Molecular interaction between Etoposide and human PARP-I.

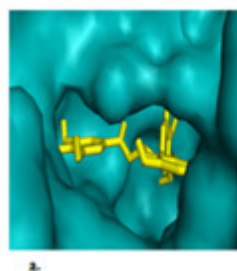

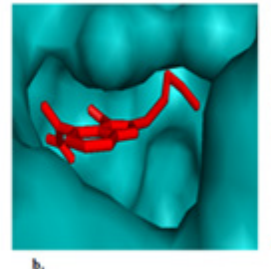

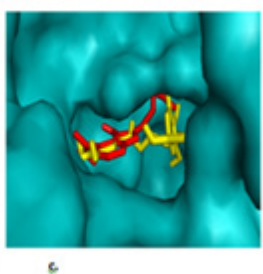

Figure 4 The binding poses of the inhibitors. (a) Stick representation of $+(-)$ gallocatechin (yellow) showing it inhibitory occupancy in the catalytic domain of PARP-I. (b) Stick representation of co-crystalized inhibitor (FSU) (red) in the catalytic domain of PARP-I, (c) a and b showing the inhibitory occupancy of $+(-)$ gallocatechn (yellow) in the catalytic domain over the co-crystalized compound (FSU)red.

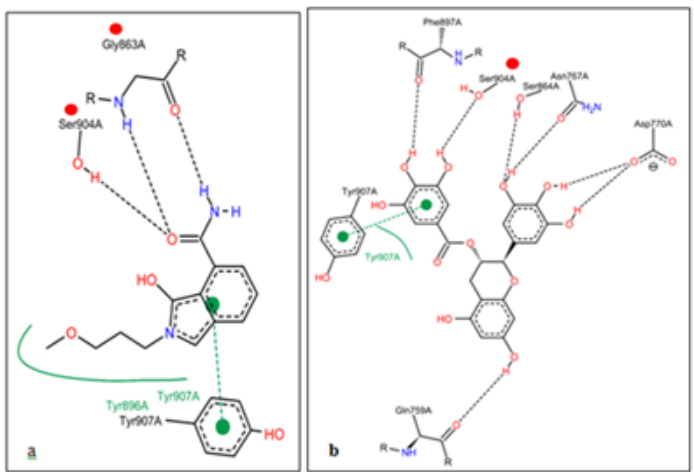

Figure 5 Showing the two-dimensional representation of molecules and their hydrogen bonding network and -stacking. (a) Showing cocrystalized inhibitor (FSU) binding mode of inhibitor of nicotinamide binding pocket as reported via hydrogen bonding network with the amino acid residues (red balls) and -stacking with the aromatic amino acid residue (green dotted line). (b) Showing gallocatechin binding modes of inhibition of nicotinamide binding pocket via hydrogen bonding with SER904 (red ball) and -stacking TYR907 (green dotted line) which has been reported.

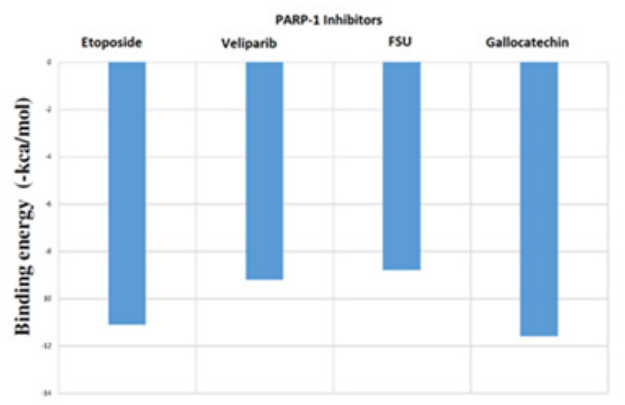

Figure 6 Bar chart showing the binding energy/affinity of Etoposide,Veliparib, Co-crystalized inhibitor (FSU) and Gallocatechin. 
Meanwhile, the anti-neoplastic potency of green tea has been clumsily overemphasized with little information about it active pharmaceutical ingredient and target chemistry [28]. With PARP-1 being a valid target for anti-cancer drug development, it only makes sense to justify that GC ( 4\%) may greatly accounts for the plants anti-neoplastic property [29]. From Figure 4c, GC fit into a region in the enzyme's active site where substrate $(\mathrm{NAD}+)$ usually binds with a potential of accurately blocking enzyme substrate from assessing the site, powering the enzyme for the cell survival mechanism.

\section{Conclusion}

By and large, we have been able to show the binding mode and the order of chemical interaction of GC within the PAPR-1 orthostheric site. Much more importantly, we justifiably predict robust binding kinetics, suitable for PARP-1 antagonism with respect to relevant FDA-approved inhibitors. With the ever increasing morbidity and mortality burdens imposed by SCLC, GC may be, in the nearest future, a single therapy with better inhibitory potency on PARP-1 derived SCLC. Meanwhile, our observations here are still subject to further confirmatory approaches that probe the atomistic, cellular and organism-wide interactions. As well, the polyphenols compounds that are not drug-like (Lipinski's RO5) can be optimized to explore their pharmacologic efficacy.

\section{Acknowledgements}

None.

\section{Conflicts of interest}

None.

\section{References}

1. Natasha R. Neuroendocrine Tumors of the Lung: An update. Arch Pathol Lab Med. 2010;134(11):1628-1638.

2. Van Meerbeeck JP, Fennell DA, De Ruysscher DK. Small cell lung cancer. Lancet. 2010;378(9804):1741-1755.

3. Byers LA, Rudin CM. Small cell lung cancer: where do we go from here? Cancer. 2015;121(5):664-672.

4. Govindan R, Page N, Morgensztern D, et al. Changing epidemiology of small-cell lung cancer in the United States over the last 30 years: analysis of the surveillance, epidemiologic, and end results database. $J$ Clin Oncol. 2006;24(28):4539-4544.

5. Byers LA, Wang J, Nilsson MB, et al. Proteomic profiling identifies dysregulated pathways in small cell lung cancer and novel therapeutic targets including PARP1. Cancer Discov. 2010;2(9):798-811.

6. Schiewer MJ, Knudsen KE. Transcriptional roles of PARP1 in cancer. Mol Cancer Res. 2014;12(8):1069-1080.

7. D'Amours D, Desnoyers S, D'Silva I, et al. Poly(ADP-ribosyl) ation reactions in the regulation of nuclear functions. Biochem $J$. 1999;342(2):249-268.

8. Kim MY, Zhang T, Kraus WL. Poly(ADP-ribosyl)ation by PARP-1: 'PAR- laying' NAD+ into a nuclear signal. Genes Dev. 2005;19(17):1951-1967.

9. Kraus WL, Lis JT. PARP goes transcription. Cell. 2003;113(6):677-683.

10. Simbulan-Rosenthal CM, Rosenthal DS, Boulares AH, et al Regulation of the expression or recruitment of components of the DNA synthesome by poly(ADP-ribose) polymerase . Biochemistry. 1998;37(26):9363-9370.
11. Simbulan-Rosenthal CM, Rosenthal DS, Luo R, et al. PARP-1 binds E2F-1 independently of its DNA binding and catalytic domains, and acts as a novel coactivator of E2F-1-mediated transcription during re-entry of quiescent cells into S phase. Oncogene. 2003;22(52):8460-8471.

12. Cohen-Armon M, Visochek L, Rozensal D, et al. DNA-independent PARP-1 activation by phosphorylated ERK2 increases Elk1 activity:a link to histone acetylation. Mol Cell. 2007;25(2):297-308.

13. Malanga M, Althaus F. The role of poly(ADP-ribose) in the DNA damage signaling network. Biochem Cell Biol. 2005;83(3):354-364.

14. Caldecott KW. XRCC1 and DNA strand break repair. DNA Repair. 2003;2(9):955-969.

15. El-Khamisy SF, Masutani M, Suzuki H, et al. Requirement for PARP-1 for the assembly or stability of XRCC1 nuclear foci at sites of oxidative DNA damage. Nucleic Acids Res. 2003;31(19):5526-5533.

16. Lan L, Nakajima S, Oohata Y, et al. In situ analysis of repair processes for oxidative DNA damage in mammalian cells. Proc Natl Acad Sci USA. 2004;101(38):13738-13743.

17. Okano S, Lan L, Tomkinson AE, et al. Translocation of XRCC1 and DNA ligase IIIalpha from centrosomes to chromosomes in response to DNA damage in mitotic human cells. Nucleic Acids Res. 2005;33(1):422-429.

18. Gassman NR, Stefanick DF, Kedar PS, et al. Hyperactivation of PARP Triggers Nonhomologous End-Joining in Repair-Deficient Mouse Fibroblasts. PLoS ONE. 2012;7(11):e49301.

19. Horton JK, Stefanick DF, Wilson SH. Involvement of poly(ADP-ribose) polymerase activity in regulating Chk1-dependent apoptotic cell death. DNA Repair (Amst). 2015;4(10):1111-1120.

20. Satoh MS, Lindahl T. Role of poly(ADP-ribose) formation in DNA repair. Nature. 1991;356(6367):356-358.

21. Heacock ML, Stefanick DF, Horton JK, et al. Alkylation DNA damage in combination with PARP inhibition results in formation of S-phase-dependent double-strand breaks. DNA Repair (Amst). 2010;9(8):929-936.

22. Chitranshi N, Gupta S, Tripathi PK, et al. New molecular scaffolds for the design of Alzheimer's acetylcholinesterase inhibitors identified using ligand- and receptor-based virtual screening. Medicinal Chemistry Research. 2013;22(5):2328-2345.

23. Lipinski CA. Lead- and drug-like compounds: the rule-of-five revolution. Drug discovery today. Technologies. 2004;1(4):337-341.

24. Stierand K, Maaß P, Rarey M. Molecular Complexes at a Glance: Automated Generation of two-dimensional Complex Diagrams. Bioinformatics. 2006;22(14):1710-1716.

25. Fricker P, Gastreich M, Rarey M. Automated Generation of Structural Molecular Formulas under Constraints. Journal of Chemical Information and Computer Sciences. 2004;44(3):1065-1078.

26. Ferraris DV. Evolution of poly(ADP-ribose) polymerase-1 (PARP-1) inhibitors. From concept to clinic. J Med Chem. 2010;53(12):4561-4584.

27. Kinoshita T, Nakanishi I, Warizaya M, et al. Inhibitor-induced structural change of the active site of human poly(ADP-ribose) polymerase. FEBS Lett. 2004;556(1-3):43-46.

28. https://medlineplus.gov/druginfo/meds/a684055.html

29. Khan N, Mukhtar H. Tea polyphenols for health promotion. Life Sci. 2007;81(7):519-533. 